\title{
LA INTELIGENCIA EMOCIONAL: MÉTODOS DE EVALUACIÓN EN EL AULA
}

\author{
Natalio Extremera Pacheco y Pablo Fernández Berrocal \\ Universidad de Málaga
}

\section{INTRODUCCIÓN}

Los educadores observan a diario que sus alumnos además de diferenciarse en su nivel académico, también difieren en sus habilidades emocionales. Estas diferencias afectivas no han pasado desapercibidas ni para sus padres, ni para el resto de compañeros de clase, ni tampoco para la ciencia. En la última década, la ciencia está demostrando que este abanico de habilidades personales influye de forma decisiva en la adaptación psicológica del alumno en clase, en su bienestar emocional e, incluso, en sus logros académicos y en su futuro laboral. Una de las líneas de investigación y estudio que hace énfasis en el uso, comprensión y regulación de los estados de ánimo es el campo de estudio de la inteligencia emocional. Desde las teorías de la inteligencia emocional se resalta que nuestras capacidades de percepción, comprensión y regulación emocional son de vital importancia para la adaptación a nuestro entorno y contribuyen sustancialmente al bienestar psicológico y al crecimiento personal, independientemente del nivel cognitivo o el rendimiento académico del alumnado (Salovey y Mayer, 1990; Mayer y Salovey, 1997). Uno de los modelos que mejor ha integrado estas premisas y está abordando el estudio científico de la inteligencia emocional es el propuesto por Mayer y Salovey (Mayer y Salovey, 1997; Mayer, Caruso y Salovey, 1999). Para estos autores la inteligencia emocional (IE) es:

"La habilidad para percibir, valorar y expresar emociones con exactitud, la habilidad para acceder y/o generar sentimientos que faciliten el pensamiento; la habilidad para comprender emociones y el conocimiento emocional y la habilidad para regular las emociones promoviendo un crecimiento emocional e intelectual' (Mayer y Salovey, 1997).

Las habilidades integradas en este modelo nos parecen de suma importancia y creemos que deben ser habilidades esenciales de obligada enseñanza en la escuela (Fernández-Berrocal y Extremera, 2002). Sin embargo, debido en parte a la confusión terminológica y a la proliferación de libros sin demasiado rigor científico que han sido publicados tras el best-seller de Goleman (Goleman, 1995; 1998), ni los investigadores ni los educadores han tenido claro qué herramientas de evaluación existen para obtener un perfil emocional de sus alumnos. A nuestro parecer, la evaluación de la IE en el aula supone una valiosa información para el docente en lo que respecta a conocimiento del desarrollo afectivo de los alumnos e implica la obtención de datos fidedignos que marquen el punto de inicio en la enseñanza transversal. El propósito de este artículo es exponer los métodos de evaluación existentes que han abordado la medición de la inteligencia emocional desde una perspectiva rigurosa. Posteriormente, se detallarán los diferentes procedimientos evaluativos que permiten obtener un buen indicador de la IE del alumnado, indicando tanto las ventajas como las limitaciones de los diferentes métodos. 
En el ámbito educativo se han empleado tres enfoques evaluativos de la IE:

?? El primer grupo incluye los instrumentos clásicos de medidas basados en cuestionarios y auto-informes cumplimentados por el propio alumno;

?? el segundo grupo reúne medidas de evaluación de observadores externos basadas en cuestionarios que son rellenados por compañeros del alumno o el propio profesor; $y$

?? el tercer grupo agrupa las llamadas medidas de habilidad o de ejecución de IE compuesta por diversas tareas emocionales que el alumno debe resolver.

\section{INSTRUMENTOS CLÁSICOS DE EVALUACIÓN DE INTELIGENCIA EMOCIONAL: CUESTIONARIOS, ESCALAS Y AUTO-INFORMES}

Este método de evaluación ha sido el más tradicional y utilizado en el campo de la psicología. A través de los cuestionarios se han obtenido perfiles en variables de personalidad como extraversión, neuroticismo, se han evaluado aspectos emocionales como empatía o autoestima y se han obtenido medidas sobre otros factores más cognitivos como pensamiento constructivo o estrategias de afrontamiento. De la misma forma, la utilización de escalas y cuestionarios está mostrando su utilidad en el campo de la IE y el manejo efectivo de nuestras emociones.

En la mayoría de los casos, estos cuestionarios están formados por enunciados verbales cortos en los que el alumno evalúa su IE mediante la propia estimación de sus niveles en determinadas habilidades emocionales a través de una escala Likert que varía desde nunca (1) a muy frecuentemente (5). Este indicador se denomina "índice de inteligencia emocional percibida o auto-informada" y revela las creencias y expectativas de los alumnos sobre si pueden percibir, discriminar y regular sus emociones.

No obstante, algunos autores como Mayer y Salovey conciben la inteligencia emocional como una inteligencia genuina y consideran deficiente una evaluación exclusivamente basada en cuestionarios de papel y lápiz. Estos autores consideran que los cuestionarios de IE pueden verse afectados por los propios sesgos perceptivos de la persona y, además, también es posible la tendencia a falsar la respuesta para crear una imagen más positiva. A pesar de ello, es indiscutible la utilidad de las medidas de auto-informes en el terreno emocional, sobretodo para proporcionar información sobre las habilidades intrapersonales y comportamientos autoinformados de los alumnos. Puesto que el mundo emocional es interior, uno de los métodos más eficaces para conocer al propio alumno, a pesar de sus sesgos, es preguntándole sobre cómo se siente, qué piensa, o cómo le afectan determinados sucesos que ocurren en el aula. La evaluación de la IE a través de cuestionarios es muy útil en aquellos casos en los que el educador quiere obtener un índice del ajuste emocional de los adolescentes y obtener un perfil de las carencias afectivas en determinadas áreas cuya única forma de evaluar sea a través de la propia introspección del alumno. Por ejemplo, es de gran utilidad para conocer la capacidad de discriminación emocional, la atención a las emociones positivas y negativas, el nivel de regulación afectiva, o el grado de tolerancia al estrés ante las frustraciones.

A continuación presentamos un ejemplo sobre la forma de evaluar el nivel de capacidad emocional basándonos en una escala típica de IE: 
Instrucciones: A continuación encontrará algunas afirmaciones sobre sus emociones y sentimientos. Lea atentamente cada frase y decida la frecuencia con la que Vd. cree que se produce cada una de ellas marcando con una $X$ en la casilla correspondiente.

\begin{tabular}{||l||l||l|l|l||}
\hline \multicolumn{1}{|c||}{} & \multicolumn{1}{|c|}{3} & \multicolumn{1}{|c||}{4} & \multicolumn{1}{|c||}{5} \\
\hline \hline NUNCA & RARAMENTE & ALGUNAS & CON & MUY \\
& & VECES & BASTAN & FRE \\
& & & TE & CUENTE \\
& & & FRECUEN & MENTE \\
& & & CIA & \\
\hline
\end{tabular}

\begin{tabular}{|c|c|c|c|c|c|c|}
\hline 1. & $\begin{array}{l}\text { Normalmente conozco } \text { mis sentimientos sobre las } \\
\text { personas. }\end{array}$ & 1 & 2 & 3 & 4 & 5 \\
\hline 2. & $\begin{array}{l}\text { Intento tener pensamientos positivos aunque me sienta } \\
\text { mal. }\end{array}$ & 1 & 2 & 3 & 4 & 5 \\
\hline 3. & Presto mucha atención a cómo me siento. & 1 & 2 & 3 & 4 & 5 \\
\hline
\end{tabular}

Como ya hemos comentado, en este método las personas se valoran a sí mismo en su capacidad percibida en distintas competencias y destrezas emocionales. Existen varios cuestionarios sobre IE que, aunque similares en su estructura, cada uno evalúa diferentes componentes de ella. Uno de los primeros cuestionarios es la Trait-Meta Mood Scale (TMMS). Este cuestionario ha sido uno de los más utilizados en el ámbito científico y aplicado. La escala proporciona una estimación personal sobre los aspectos reflexivos de nuestra experiencia emocional. La TMMS contiene tres dimensiones claves de la inteligencia emocional intrapersonal: Atención a los propios sentimientos (p.e. "Pienso en mi estado de ánimo constantemente'), Claridad emocional (p.e. "Frecuentemente me equivoco con mis sentimientos") y Reparación de las propias emociones (p.e. "Aunque a veces me siento triste, suelo tener una visión optimista"). La versión clásica contiene 48 ítems aunque hay versiones reducidas de 30 y de 24 ítems (Salovey, Mayer, Goldman, Turvey y Palfai, 1995, adaptación al castellano por Fernández-Berrocal, Alcaide, Domínguez, Fernández-McNally, Ramos y Ravira, 1998).

Otro instrumento similar al anterior es la escala de E de Schutte. Esta medida proporciona una única puntuación de inteligencia emocional (Schutte, Malouff, Hall, Haggerty, Cooper, Golden y Dornheim, 1998, adaptación al castellano por Chico, 1999). Sin embargo, investigaciones posteriores han encontrado que esta escala se puede dividir en cuatro subfactores: 1) Percepción emocional p.e. "Encuentro difícil entender los mensajes no verbales de otras personas"); 2) Manejo de emociones propias (p.e. "Me motivo a mi mismo imaginando un buen resultado en las tareas que voy a hacer"); 3) Manejo de las emociones de los demás (p.e. "Solucionar los problemas de los demás me divierte") y, por último, 4) Utilización de las emociones (p.e. "Cuando siento que mis emociones cambian, se me ocurren nuevas ideas"). 
Otro de los instrumentos empleados en la investigación es el inventario EQ-i de Bar-On (1997, adaptado al castellano por MHS, Toronto, Canadá). No obstante, como sus propios autores han afirmado se trata más bien de un inventario sobre una amplia gama de habilidades emocionales y sociales más que un instrumento genuino de IE. Esta medida contiene 133 ítems y esta compuesta por cinco factores generales, que se descomponen en un total de 15 subescalas: 1) inteligencia intrapersonal, evalúa las habilidades de autoconciencia-emocional, autoestima personal, asertividad, auto-actualización e independencia; 2) inteligencia interpersonal, que comprende las subescalas de empatía, relaciones interpersonales y responsabilidad social; 3) adaptación, que incluye las habilidades de solución de problemas, comprobación de la realidad y flexibilidad; 4) gestión del estrés, compuesta por las subescalas de tolerancia al estrés y control de impulsos y 5) humor general, integrada por las subescalas de felicidad y optimismo. Además, el inventario incluye 4 indicadores de validez que miden el grado con que los sujetos responden al azar 0 distorsionan sus respuestas y cuyo objetivo es reducir el efecto de deseabilidad social e incrementar la seguridad de los resultados obtenidos.

\section{MEDIDAS DE EVALUACIÓN DE INTELIGENCIA EMOCIONAL BASADA EN OBSERVADORES EXTERNOS}

Esta segunda forma de evaluar los niveles de la IE parte de un presupuesto básico: si la IE implica la capacidad para manejar y comprender las emociones de las personas que nos rodean, ¿por qué no preguntar a las personas más cercanas a nosotros sobre cómo manejamos nuestras emociones en público y la forma de afrontar los sucesos que nos ocurren en nuestra vida cotidiana? Este procedimiento se considera un medio eficaz para evaluar la inteligencia emocional interpersonal, es decir, nos indica el nivel de habilidad emocional percibida por los demás. Comúnmente son llamados instrumentos basados en la observación externa o evaluación 360ำ, en ellos se solicita la estimación por parte de los compañeros de clase o el profesor para que nos den su opinión sobre cómo el alumno es percibido con respecto a su interacción con el resto de compañeros, su manera de resolver los conflictos en el aula o bien su forma de afrontar las situaciones de estrés. Esta forma de evaluación es complementaria al primer grupo de medidas y sirve de información adicional y como medida para evitar posibles sesgos de deseabilidad social. Algunos cuestionarios, como EQ-i de Bar-On (1997) incluye un instrumento de observación externa complementario al cuestionario que debe cumplimentar el alumno. En otras ocasiones, se emplean técnicas sociométricas denominadas "peer nominations" en las que el alumnado y/o profesorado valora al resto de la clase en diferentes adjetivos emocionales y estilos de comportamiento habituales.

A continuación presentamos un ejemplo sobre la forma de evaluar el nivel de capacidad emocional interpersonal basándonos en un método de observación externa:

Instrucciones: A continuación te presentamos una serie de afirmaciones que describen formas de ser 0 comportamientos generales de las personas. Lea atentamente cada frase y valora a tu compañero en cada una de ellas.

Indica por favor el grado de acuerdo o desacuerdo con respecto a las mismas usando la siguiente escala y marcando con una $X$ en la casilla correspondiente. 


\begin{tabular}{|c||c||c||c|c||}
\hline 1 & 2 & 3 & 4 & 5 \\
\hline \hline NADA DE & ALGO DE & BASTANTE DE & MUY DE & TOTALMENTE DE \\
ACUERDO & ACUERDO & ACUERDO & ACUERDO & ACUERDO \\
\hline
\end{tabular}

\begin{tabular}{|c|c|c|c|c|c|}
\hline ¿Es capaz de entender a la gente? & & 2 & 3 & & 5 \\
\hline ¿Es una persona a la que se le pueden contar problemas personales? & 1 & 2 & 3 & 4 & 5 \\
\hline ¿Maneja bien las situaciones de conflicto o estrés? & 1 & 2 & 3 & 4 & 5 \\
\hline
\end{tabular}

Como habrá apreciado el lector, este tipo de metodología evalúa aspectos interpersonales y adolece también de algunas limitaciones. Primero, puesto que es muy difícil estar con una persona las 24 horas del día, la evaluación del observador depende de la forma de comportarse el individuo en presencia de él y, por tanto, se trata de una evaluación basada en observaciones de otra persona con sus respectivos sesgos perceptivos. Segundo, ya que es difícil que un observador esté en todas las situaciones con el alumno y es sabido que la forma de comportarnos varía en función del contexto, la información que nos proporciona esta metodología esta restringida a un contexto determinado como es el aula. Tercero, con este procedimiento es difícil obtener datos sobre las habilidades emocionales intrapersonales tales como la capacidad de conciencia emocional, atención afectiva o claridad emocional interna. No obstante, esta metodología aporta nueva información no facilitada por las medidas anteriores. A través de la evaluación por observadores externos obtenemos información muy valiosa sobre cómo perciben al alumno los demás compañeros a nivel socio-emocional y es de gran utilidad para evaluar destrezas relacionadas con las habilidades interpersonales, la falta de autocontrol y los niveles de impulsividad y de manejo emocional en situaciones de conflicto social como, por ejemplo, una pelea entre dos alumnos.

\section{MEDIDAS DE HABILIDAD DE LA INTELIGENCIA EMOCIONAL BASADAS EN TAREAS DE EJECUCIÓN}

Este último grupo de medidas surge para suplir los problemas de sesgos que presentan las dos metodologías anteriores. El objetivo de estos instrumentos es, por una parte, evitar la falsación de las respuestas por los propios sujetos en situaciones donde una imagen positiva es deseable y, por otra, disminuir los sesgos perceptivos y situacionales provocados por los observadores externos. La lógica de estos instrumentos de evaluación es de un sentido común aplastante. Si queremos evaluar si una persona es hábil o no en un ámbito, la mejor forma de hacerlo es comprobar sus habilidades. Por ejemplo, si nuestro propósito es conseguir entre los alumnos al mejor pianista del colegio para realizar una obra musical, la forma ideal para evaluar las destrezas al piano de manera rápida es darles una partitura de Beethoven y pedirles que intenten tocarla. Es decir, no se solicita al alumno que nos diga cómo cree él que toca de bien el piano o no se le pregunta a un familiar o amigo si sabe tocar el piano. La prueba requiere que muestre sus propias habilidades tocando el piano. 
En general, las medidas de habilidad consisten en un conjunto de tareas emocionales, más novedosas en su procedimiento y formato, que evalúan el estilo en que un estudiante resuelve determinados problemas emocionales comparando sus respuestas con criterios de puntuación predeterminados y objetivos (Mayer et al., 1999; Mayer, 2001). Por ejemplo, si queremos evaluar la percepción emocional se examina si el adolescente es capaz de reconocer emociones en ciertas expresiones faciales. Igualmente, si nuestro propósito es evaluar la capacidad de manejo emocional se valorará la idoneidad de las estrategias de solución de problemas seleccionadas por el alumno para resolver un conflicto interpersonal. Existen dos medidas de habilidad para evaluar la IE desde este acercamiento, el MEIS (Mayer, Caruso y Salovey, 1999) basada en el modelo de Salovey y Mayer (1990) y su versión mejorada el MSCEIT (Mayer, Salovey y Caruso, 2002; Mayer, et al., 1999). Estas medidas abarcan cuatros áreas de la inteligencia emocional propuesta en la definición de Mayer y Salovey. (Mayer y Salovey, 1997): 1) percepción emocional; 2) asimilación emocional, 3) comprensión emocional y 4) regulación afectiva. En la medida que la IE es considerada una forma de inteligencia como la inteligencia verbal, espacial o matemática, los autores consideran que puede evaluarse mediante diferentes tareas emocionales de la misma manera que el cociente intelectual $(\mathrm{Cl})$ puede evaluarse a través de las habilidades puestas en práctica, por ejemplo, en el test de inteligencia de Weschler.

A continuación presentamos un ejemplo sobre la forma de evaluar la IE basándonos en medidas de habilidad:

\section{Percepción emocional}

Instrucciones: A continuación vas a ver una serie de rostros faciales. Míralos y luego indica los sentimientos expresados en esos rostros. Indica el grado en el que crees que las emociones están presentes en cada una de las caras utilizado las emociones que te presentamos a continuación.

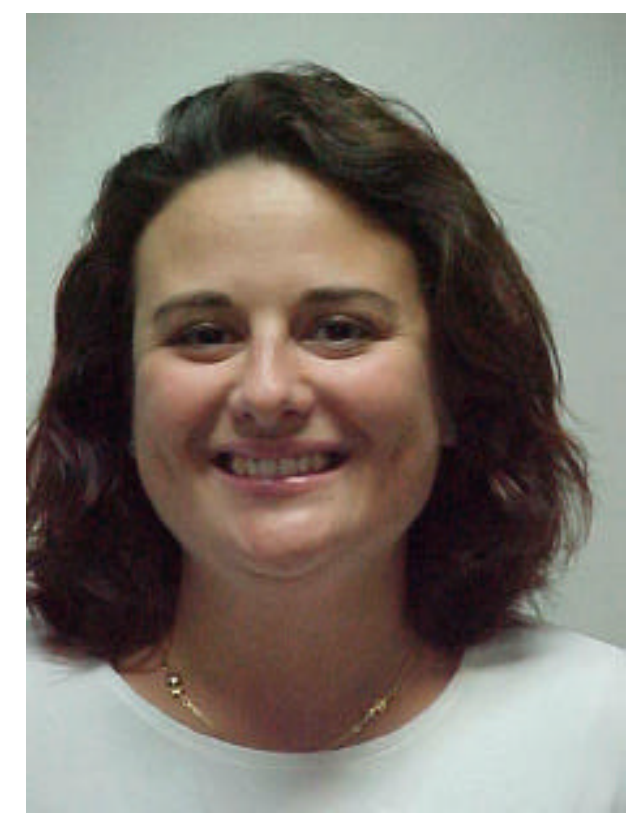




\begin{tabular}{|l|c|c|c|c|c|l|}
\hline Nada de Enfado & 1 & 2 & 3 & 4 & 5 & $\begin{array}{l}\text { Enfado } \\
\text { Extremo }\end{array}$ \\
\hline Nada de Tristeza & 1 & 2 & 3 & 4 & 5 & $\begin{array}{l}\text { Tristeza } \\
\text { Extrema }\end{array}$ \\
\hline Nada de Felicidad & 1 & 2 & 3 & 4 & 5 & $\begin{array}{l}\text { Felicidad } \\
\text { Extrema }\end{array}$ \\
\hline Nada de Miedo & 1 & 2 & 3 & 4 & 5 & $\begin{array}{l}\text { Miedo } \\
\text { Extremo }\end{array}$ \\
\hline
\end{tabular}

\section{Asimilación emocional}

Instrucciones: A continuación se te presentan una serie de situaciones cotidianas. Por favor, selecciona una respuesta para cada ítem e indica qué emoción o emociones serían útiles para resolverlas.

¿Que estado/s de ánimo sería útil cuando intentas resolver un problema difícil como, por ejemplo, un ecuación matemática?

\begin{tabular}{|l|c|c|c|c|c|}
\hline & Nada útil & & & & Muy útil \\
\hline Tensión & 1 & 2 & 3 & 4 & 5 \\
\hline Tristeza & 1 & 2 & 3 & 4 & 5 \\
\hline Diversión & 1 & 2 & 3 & 4 & 5 \\
\hline
\end{tabular}

\section{Comprensión emocional}

Instrucciones: A continuación aparecen una serie de situaciones sobre diferentes personas. Una vez hayas leído las situaciones, tu tarea consistirá en indicar cómo se deben sentir esas personas.

Alberto está cansado y se encuentra incluso estresado cuando piensa en todo los deberes que le quedan por hacer y los exámenes que tiene que estudiar. Cuando ese mismo día el profesor les manda otro trabajo y les dice que tienen que terminarlo esa misma semana, Alberto se sintió
a. saturado
b. deprimido
c. culpable
d. frustrado
e. nervioso

\section{Regulación emocional}

Instrucciones: A continuación te vamos a presentar una serie de situaciones que incluyen a varias personas. Tu tarea consiste en elegir qué acciones o estrategias emocionales serían más beneficiosas para mantener el estado de ánimo de esas personas.

Juana acaba de venir de sus vacaciones. Se siente relajada, animada y llena de energía. ¿En qué medida cada una de las siguientes acciones ayudarían a Juana a mantener esas emociones?

Acción 1: Juana empezó a hacer una lista de las cosas de casa que tenía que hacer.
1. Muy ineficaz
2. Algo ineficaz
3. Neutral
4.Algo eficaz 5.Muy eficaz

Acción 2: Empezó a pensar sobre dónde y cuándo serían sus próximas vacaciones.
1. Muy ineficaz
2. Algo ineficaz
3. Neutral
4.Algo eficaz 5.Muy eficaz

Acción 3: Decidió que lo mejor era ignorar esos sentimientos positivos y enfrentarse otra vez a la realidad.
1. Muy ineficaz
2. Algo ineficaz
3. Neutral
4.Algo eficaz 5.Muy eficaz 
Acción 4: Llamó a una amiga para contarle sus vacaciones.
1. Muy ineficaz
2. Algo ineficaz
3. Neutral
4.Algo eficaz 5.Muy eficaz

Este grupo de medidas también presenta sus limitaciones:

?? Se trata de una forma de evaluación tan novedosa que todavía requiere mejorar sus propiedades psicométricas.

?? Como ocurre con aquellos instrumentos que presentan una situación concreta a los sujetos que deben resolver, las pruebas son muy contextuales y deben adaptarse en función de la población que se vaya a evaluar. Por ejemplo, aunque ya se han desarrollado el MEIS y el MSCEIT, estas pruebas son para personas con una edad superior a 17 años tanto por las situaciones emocionales que se presentan como por la capacidad de comprensión que requieren. Actualmente, se está desarrollando una medida de habilidad con una mayor aplicabilidad al aula y adaptada a las capacidades cognitivas de niños y adolescentes en la que, además, se presentan situaciones de conflicto interpersonal, de frustraciones o de manejo afectivo más relacionadas con el contexto educativo.

?? Por último, otra de las limitaciones de estos cuestionarios es que requieren de mayor tiempo para su cumplimentación en comparación con las medidas anteriores, lo cual implica que pueden encontrarse sesgos en las respuestas ocasionadas por el cansancio del alumnado. Todas estas limitaciones están siendo tratadas en las nuevas versiones que se están desarrollando.

No obstante, las ventajas de esta nueva forma de medición son indudables, sobretodo en combinación con algunas de las medidas anteriores. A través de este tipo de instrumentos obtenemos indicadores de ejecución en habilidades emocionales concretas que pueden ser educadas y entrenadas posteriormente. Nos permite, además, evitar sesgos perceptivos y son más difíciles de distorsionar, debido a su formato, cuando el alumno trata de ofrecernos una imagen más positiva de sí mismo.

\section{CONCLUSIÓN}

En este artículo hemos querido resaltar la importancia que tiene la evaluación de la inteligencia emocional en el ámbito educativo y dar a conocer a los educadores las últimas herramientas de evaluación existentes.

Para concluir nos gustaría resaltar tres aspectos que consideramos fundamentales para el futuro de la investigación en este campo.

En primer lugar, imaginemos en un alarde de optimismo que la comunidad educativa estuviera convencida de que la inteligencia emocional debiera ser uno de los objetivos básicos a perseguir por el 
sistema educativo, pero no dispusiéramos de la tecnología adecuada para hacerlo. ¿Qué haríamos? Sería difícil trabajar con la inteligencia emocional, ya que resultaría casi imposible valorar, por ejemplo, si los programas de mejora que estuviéramos utilizando en el aula están funcionando o no. Por ello, es tan relevante avanzar en el desarrollo de instrumentos que evalúen la inteligencia emocional con la mayor precisión y validez posible y, a su vez, darle la máxima difusión en el ámbito educativo.

En segundo, los instrumentos para evaluar la inteligencia emocional que hemos descrito no evalúan todos los aspectos posibles de la inteligencia emocional, captan sólo una parte de lo que conceptualmente entendemos por ella (en especial, los aspectos intrapersonales). Aunque parece, por los estudios existentes, que la parcela evaluada es lo suficientemente representativa.

En este sentido, queda el reto de construir nuevos instrumentos que evalúen otros aspectos de la inteligencia emocional, aún no bien representados en las medidas existentes como los aspectos interpersonales.

Por último, la nueva generación de instrumentos deberá ser lo más realista posible y captar la idiosincrasia de cada cultura ya que, a pesar del esfuerzo de adaptación, los actuales han sido generados en el ámbito anglosajón.

\section{REFERENCIAS}

(Se han añadido otras referencias relacionadas con la evaluación de la inteligencia emocional que pueden ser de interés para el lector).

Bar-On, R. (1997) The Emotional Quotient Inventory (EQ-i): A test of emotional intelligence. Toronto: MultiHealth Systems.

Caruso, D. R., Mayer, J. D, y Salovey, P. (2002) Relation of an ability measure of emotional intelligence to personality. Journal of Personality Assessment 79 (2), 306-320.

Chico, E. (1999). Evaluación psicométrica de una escala de Inteligencia Emocional. Boletín de Psicología, $62,65-78$.

Extremera, N. y Fernández-Berrocal, P. (2002) Relation of perceived emocional intelligence and healthrelated quality of life of middle-aged women. Psychological Reports, 91, 47-59.

Fernández-Berrocal, P., Alcaide, R., Domínguez, E., Fernández-McNally, C., Ramos, N. S., y Ravira, M. (1998) Adaptación al castellano de la escala rasgo de metaconocimiento sobre estados emocionales de Salovey et al.: datos preliminares. Libro de Actas del V Congreso de Evaluación Psicológica. Málaga.

Fernández-Berrocal, P. y Extremera, N. (2002) La inteligencia emocional como una habilidad esencial en la escuela. Revista Iberoamericana de Educación, 29, 1-6.

Fernández-Berrocal, P. y Ramos, N. (2002) Corazones Inteligentes. Barcelona: Kairós.

Fernández-Berrocal, P., Ramos, N., y Orozco, F. (1999) La influencia de la inteligencia emocional en la sintomatología depresiva durante el embarazo. Toko-Ginecología Práctica, 59, 1-5.

Fernández-Berrocal, P., Ramos, N., y Extremera, N. (2001) Inteligencia emocional, supresión crónica de pensamientos y ajuste psicológico. Boletín de Psicología, 70, 79-95. 
Fernández-Berrocal, P., Salovey, P., Vera, A., Ramos, N., y Extremera, N. (2001) Cultura, inteligencia emocional percibida y ajuste emocional: un estudio preliminar. Revista Electrónica de Motivación y Emoción, 4, 1-15.

Goldman, S. L., Kraemer, D. T., y Salovey, P. (1996) Beliefs about mood moderate the relationship of stress to illness and symptom reporting. Journal of Psychosomatic Research, 41, 115-128.

Goleman, D. (1995) Emotional intelligence. New York: Bantam.

Goleman, D. (1998) Working with emotional intelligence. New York: Bantam.

Mayer, J. D. y Salovey, P. (1997) What is emotional intelligence? En P. Salovey y D. Sluyter (Eds). Emotional Development and Emotional Intelligence: Implications for Educators (pp. 3-31). New York: Basic Books.

Mayer, J., Caruso, D. y Salovey, P. (1999) Emotional intelligence meets traditional standards for an intelligence. Intelligence, 27, 267-298.

Mayer, J. D., Salovey, P., y Caruso, D. R. (2002) Mayer-Salovey-Caruso Emotional Intelligence Test (MSCEIT) Item Booklet. Toronto, Canada: MHS Publishers.

Mayer, J. D., Salovey, P., Caruso, D. R., y Sitarenios, G. (2001). Emotional intelligence as a standard intelligence: A Reply. Emotion, 1, 232-242.

Salovey, P. (2001) Applied emotional intelligence: Regulating emotions to become healthy, wealthy, and wise. Emotional intelligence and intimate relationships. En J. Ciarrochi, J. P. Forgas, y J. D. Mayer (Eds.) Emotional Intelligence and Everyday Life. New York: Psychology Press. Pp.168-184.

Salovey, P., Bedell, B., Detweiler, J. B., y Mayer, J. D. (1999) Coping intelligently: Emotional intelligence and the coping process. En C. R. Snyder (Eds), Coping: The psychology of what works. New York: Oxford University Press. Pp.141-164.

Salovey, P., Bedell, B., Detweiler, J.B., y Mayer, J. D. (2000) Current directions in emotional intelligence research. En M. Lewis y J. M. Haviland-Jones (Eds.), Handbook of Emotions (2 ${ }^{\text {nd }}$ ed.). New York: Guilford Press. Pp. 504-520.

Salovey, P. y Mayer, J. D. (1990) Emotional intelligence. Imagination, Cognition, and Personality, 9,185-211. Salovey, P., Mayer, J. D., Goldman, S. L., Turvey, C., y Palfai, T. P. (1995) Emotional attention, clarity, and repair: Exploring emotional intelligence using the Trait Meta-Mood Scale. En J. W. Pennebaker (Ed.), Emotion, Disclosure, \& Health. Washington: American Psychological Association. Pp. 125-151.

Salovey, P., Stroud, L., Woolery, A., y Epel, E. (2002) Perceived emotional intelligence, stress reactivity and symptom reports: Furthers explorations using the Trait Meta-Mood Scale. Psychology and Health, 17, 611-627.

Salovey, P., Woolery, A., y Mayer, J. D. (2001) Emotional intelligence: Conceptualization and measurement. En G.J.O. Fletcher \& M. S. Clark (Eds). Blackwell Handbook of Social Psychology: Interpersonal Processes. Malden, MA: Blackwell Publishers. Pp.279-307.

Schutte, N. S., Malouff, J. M., Hall, L. E., Haggerty, D. J., Cooper, J. T., Golden, C. J., y Dornheim, L. (1998) Development and validation of a measure of emotional intelligence. Personality and Individual Differences, 25, 167-177.

Trinidad, D. R., y Johnson, C. A. (2002) The association between emotional intelligence and early adolescent tobacco and alcohol use. Personality and Individual Differences, 32, 95-105. 
Nota. Para obtener los test EQ-i de Bar-On y el MSCEIT de Salovey es necesario ponerse en contacto con MHS Publishers (www.mhs.com). El TMMS en castellano puede solicitarse a los autores de este artículo por correo eletrónico (berrocal@uma.es; nextremera@uma.es). 


\title{
Contactar
}

Revista lberoamericana de Educación

\author{
Principal OEI
}

